\title{
Working Range Assessment for Interventional Guidance Using Two Electromagnetic Sensors
}

\author{
Chia-Hsiang Wu, Mei Yun Su, Cheng-Chien Chang, and Ting-Sheng Lin
}

\begin{abstract}
Electromagnetic trackers have been used extensively in image-guided surgery and therapy. During the procedure, it is essential to evaluate the effective working range of the tracking system, because the accuracy could be influenced by the environment and manner in which the procedure is conducted. We assume that the guidance is provided by two electromagnetic sensors attached at the distal ends of two endoscopes in order to achieve end-to-end coaptation of the human inner tubular structure. During the procedure, the sensors become very close to help surgeons find the desired path inside the body; as a result, the sensors can potentially cause distortion that adversely affects the accuracy of the tracking system. Thus, it is necessary to determine the working range of the tracking system. The range was evaluated by two methods: static measurement and dynamic measurement. Static measurement determines the range by placing the sensors in several positions and collecting data when the sensors are stationary, whereas dynamic measurement allows movement of the sensors. The experimental results show that these two methods are useful in determining the effective working range.
\end{abstract}

Index Terms-Endoscope, electromagnetic tracking system, interventional guidance.

\section{INTRODUCTION}

Electromagnetic tracking systems have been used extensively in image-guided surgery. They provide the position and orientation of the instruments to help the surgeon understand the geometrical relationships between the patient and the medical devices. The major advantage of these systems is that they work when the sensor is occluded; however, the working range may be limited due to ferrous metals or conductive materials within the working volume of the tracking system. In this study, we assume that the procedure was guided by two electromagnetic sensors attached at the distal ends of two endoscopes in order to achieve end-to-end coapt for the severance of the uterine tube During the procedure, the sensors should become closer and closer; the distal tips could eventually contact each other to help the surgeons to find the desired path inside the body. That is, sensors within the surgical field could cause distortion that adversely affects the accuracy of the tracking system. Thus, it is necessary to know how sensor accuracy is

Manuscript received May 10, 2013; revised July 16, 2013. This work was supported by the National Science Council of the Republic of China (Taiwan) under grant no. NSC 101-2221-E-214 -038.

C.-H. Wu, M. Y. Su, and T.-S. Lin are with the Department of Biomedical Engineering, I-Shou University, Kaohsiung, Taiwan (e-mail: chwu@ isu.edu.tw).

C.-C. Chang is with the Department of Urology, Sin-Lau Hospital, Tainan. affected when the distance between the sensors is very short.

To investigate how items cause distortion of an electromagnetic tracking system, several studies have been conducted. The distortion generally correlates with the distance between the item and the sensor, and items that are closer to the sensor can have larger impacts. These items include surgical instruments, ultrasound probes, and surgical tables. Schico et al. [3] simulated the conditions in the operating room and evaluated the possible distortions of electromagnetic tracking systems caused by three different kinds of surgical instruments. They placed the surgical instruments at different distances from the sensor to evaluate the distortion and found that the distortion was not significant. Hastenteufel et al. [1] investigated the distortions of electromagnetic sensors caused by 3D ultrasound probes. They found that the 3D probes caused greater distortions of electromagnetic sensors than 2D probes, and they suggested that the interference should be checked carefully. In an operating room, surgical tables can also be a source of distortion, especially for procedures where the sensor is located in close proximity to the table [8].

In addition to evaluating the effect of specific items, it is also possible to evaluate overall clinical environments. Yaniv et al. [4] identified the factors that influence the utility of an electromagnetic tracking system by assessing three clinical environments, including an interventional radiology suite, a CT suite, and apulmonology suite. Their experiments show that these systems can be used successfully in these environments. Based on the research findings, the extent of the distortion that occurs depends on the kinds of instruments that are used and on the environment in which they are used. Moreover, in an electromagnetic tracking system, a field generator is necessary to track the electromagnetic sensor. Thus, the accuracy and precision of field generators in an interventional radiology setting have been investigated [6]. Accuracy and precision were assessed using a machined base plate to measure errors in position and orientation. A similar study was conducted for ultrasound-guided intervention [7]. The researchers concluded that the influence of the attachment of an ultrasound probe to the field generator is not critical in most cases.

In this study, we consider a procedure guided by two electromagnetic sensors attached at the distal ends of two endoscopes. The purpose of this study is to find the working range between the sensors and the transmitter, so that the sensed data within the range are considered reliable. In the next section, we introduce the experimental setup and evaluation methods. Section III gives the experimental results, followed by conclusions. 


\section{METHOD}

\section{A. Experimental Setup}

The 3D Guidance trak STAR electromagnetic tracking system (Ascension Technology, USA), which is based on pulsed direct-current (DC) technology, was used in the experiments. The pulsed DC magnetic fields were generated by a mid-range transmitter (field generator) for tracking over medium range. Three sensors were used: two Model 1806 DOF (degree-of-freedom) sensors and one Model 800 6DOF sensor. Their diameters were $2 \mathrm{~mm}$ and $8 \mathrm{~mm}$, respectively. The $2 \mathrm{~mm}$ sensors were assumed to be located at the distal tips of the endoscopes; the $8 \mathrm{~mm}$ sensor, referred to as the reference sensor in this paper, served as the position reference for dynamic measurement (Section II-C).The data were transmitted to a host computer via USB cable at a default refresh rate of $240 \mathrm{~Hz}$.

The sensor configuration is shown in Fig. 1. The locations of the sensors, as indicated by the red circles, were fixed in a custom-made plate. The right one is the reference sensor, and the left two are the Model 180 sensors located in a plastic tube inside the plate. The Model 180 sensors were placed head-to-head in close proximity to simulate the maximum possible error. Although the two sensors should be placed in the endoscopes, a larger space is needed, and it is difficult to have two endoscopes head-to-head and fixed during the experiment. Hence, we used the configuration shown in Fig. 1 instead of real endoscopes in order to fix and keep the sensors as close as possible in the experiments.

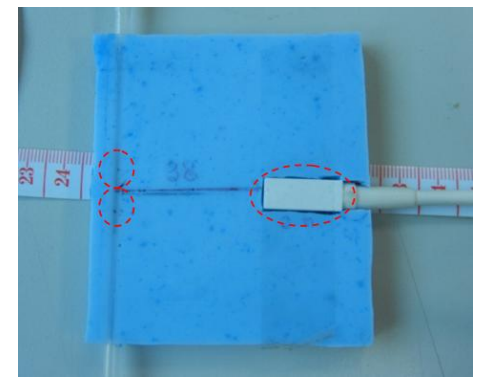

Fig. 1. Sensor configuration.

\section{B. Static Measurement}

We first performed static measurement, which means that we collected data only when the sensors were stationary. The errors were measured as the Euclidean distance $d^{t}$ between the two Model 180 sensors at time $t$

$$
d^{t}=\left\|\overline{p_{1}^{t} p_{2}^{t}}\right\|
$$

where $p_{1}^{t}=\left(x_{1}^{t}, y_{1}^{t}, z_{1}^{t}\right)$ and $p_{2}^{t}=\left(x_{2}^{t}, y_{2}^{t}, z_{2}^{t}\right)$ are the positions of the two sensors reported by the tracking system. The sensors were fixed in the plate so the distance is constant, but the measured distance $d^{t}$ actually depends on the range between the plate and the transmitter. The accuracy was determined based on variations of $d^{t}$ with respect to the distance between the plate and the transmitter.

Given a circle centered at the transmitter and having radius $R \mathrm{~mm}$, we collected data every $D \mathrm{~mm}$ along the radius from the transmitter, resulting in a total of $n=\lceil R / D\rceil$ positions to be sampled (the 0 -mm was ignored). Each position was sampled for $T$ seconds. The standard deviation, $s_{i}$, of the distance sequence $\left\{d^{t, i}\right\}$ at the $i$ th position based on the reported data $\left\{p_{1}^{t, i}\right\}$ and $\left\{p_{2}^{t, i}\right\}$ were calculated for $t=1 / H$, $2 / H, \ldots, H T$ and $i=1,2,3, \ldots, n$, where $H$ is the refresh rate:

$$
s_{i}=\sqrt{\frac{1}{H T} \sum_{t=1}^{H T}\left(d^{t, i}-m_{i}\right)^{2}}
$$

The standard deviation $s_{i}$ represents the variation of $d^{t, i}$, i.e., stability of the data.

\section{Dynamic Measurement}

The second method is called dynamic measurement. It allows movement of the sensor during data collection, so the error was evaluated continuously rather than collecting data at certain positions. If the sensors are moving, it is hard to identify the sampling positions; thus, we attached the reference sensor to capture the actual positions of the two sensors. We also moved the sensors along the radius of the circle centered at the transmitter. After obtaining the sequence $\left\{d^{t}\right\}$, we estimated the variation of the measured distance between two Model 180 sensors by the magnitude of approximated derivatives defined by the central difference of the sequence:

$$
\left|d^{\prime}(t)\right| \cong\left|\frac{d(t+h)-d(t-h)}{2 h}\right|
$$

where $h$ is the time interval equal to the reciprocal of the refresh rate and $d(t)=d^{t}$. Equation (3) gives the variation with respect to time. If the speed of the sensors is a constant $k$, the Euclidean distance $f(p)$ between the two sensors with respect to space (the distance $p$ between the two sensors and the transmitter) is

$$
f(p)=d\left(\frac{p}{k}\right)
$$

Hence, the amount of variation with respect to space can be evaluated.

In practice, a constant speed can only be achieved by specific devices, so the speed is unavailable in our design. In our sensor configuration, the distance $p$ can be evaluated by the reference sensor, and $d(t)$ can be associated with distance $p$.Thus, we denote $p_{t}$ as the distance $p$ measured at time $t$, and the amount of variation with respect to space is approximated by

$$
\left|f^{\prime}\left(p_{t}\right)\right| \propto\left|\frac{d(t+h)-d(t-h)}{2}\right| .
$$

\section{EXPERIMENTAL RESULTS}

For the static measurement, the parameters are $R=500 \mathrm{~mm}$, $D=50 \mathrm{~mm}, \quad T=5$ seconds, and $H=240 \mathrm{~Hz}$. The standard deviation of the distance between the two sensors versus the distance between the plate and the transmitter is shown in Fig. 2. The standard deviation began to increase when the sensors were about $250 \mathrm{~mm}$ from the transmitter. To evaluate whether the increase of the standard deviation indicates the tendency of deviation from the actual values, an actual distance of 8.9 $\mathrm{mm}$ between the two sensors was obtained by turning off the sensors alternately. This actual distance is compared to the 
mean distance between sensors based on the reported data. It can be seen in Fig. 2 b) that the mean distance deviates from the actual distance when the plate-to-transmitter distance is larger than $250 \mathrm{~mm}$. This result is consistent to the observation based on $s_{i}$, which validates the static measurement, is likely to be useful in determining the working range based on changes in standard deviation.

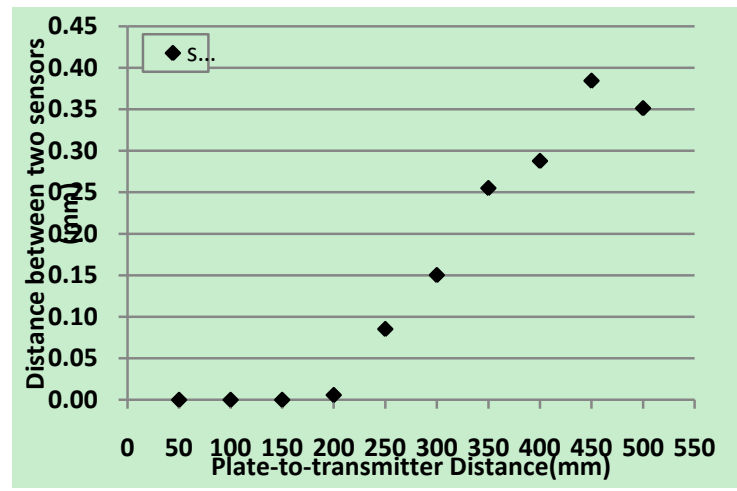

a)

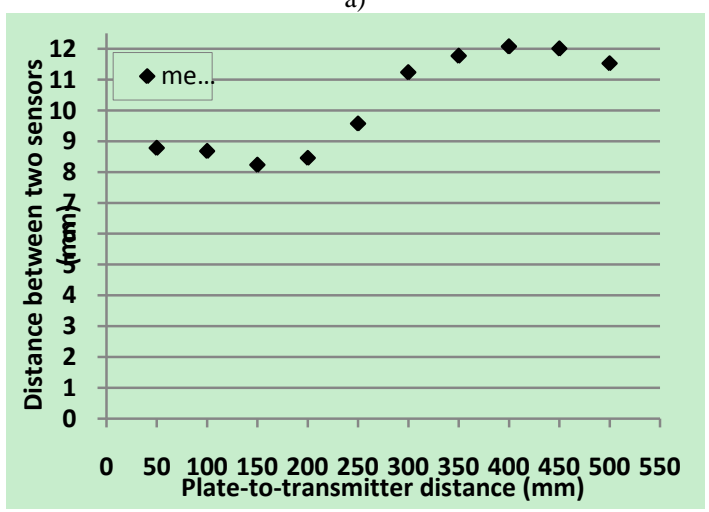

b)

Fig. 2. Static mesaurement. a) Standard deviation and b)mean of the distance between two sensors versus the distance between the plate and the transmitter.

In the dynamic measurement, the reference sensor was added to capture the motion of the Model 180 sensors and report the actual distance between the plate and the transmitter. We began by experimenting in the range of 0 to $1200 \mathrm{~mm}$. This range, in fact, exceeded the translation range listed in the specification sheet of the tracking system; it is a useful evaluation, because the surgeon may not be aware of the effective range during a procedure. According to Fig. 3 a), the reported distance between two sensors tended to become instable when the plate was away from the transmitter. Fig. 3 b) shows the results in closer range from 0 to $500 \mathrm{~mm}$. The distance between two sensors is considered as stable within about $240 \mathrm{~mm}$ (actual distance of $8.9 \mathrm{~mm}$ plus or minus $1 \mathrm{~mm}$ ). In the dynamic experiment, we did not collect data in a stationary position; thus, the variation cannot be evaluated by the standard deviation as was done in the static experiment. Finite differencing in (5) was used as an alternative means of determining the variation, as shown in Fig. 3 c) and Fig. 3 d), ranging from $0-1200 \mathrm{~mm}$ and $0-500 \mathrm{~mm}$, respectively. It can be seen that the finite difference is useful for larger range; i.e., the variation becomes larger after $500 \mathrm{~mm}$. However, variation is less significant in Fig. 3 d). Thus, the dynamic measurement based on finite difference is also useful in reflecting the effective working range, but it -sless sensitive than the static experiment for a shorter range.

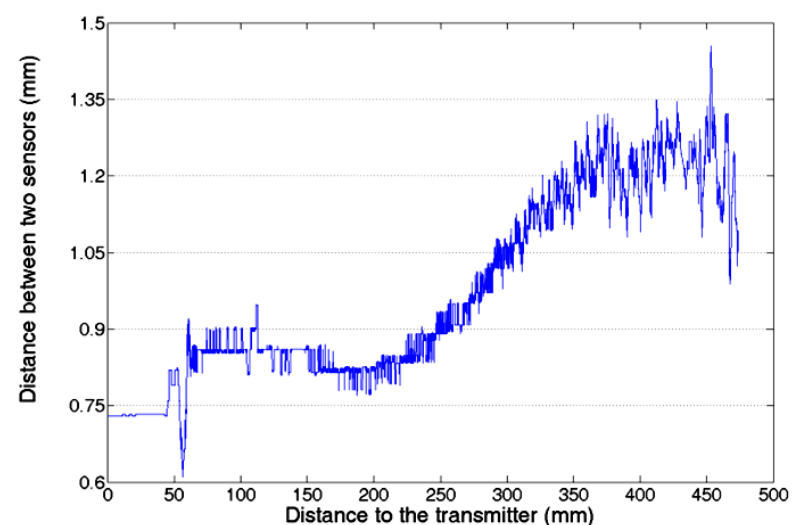

a)

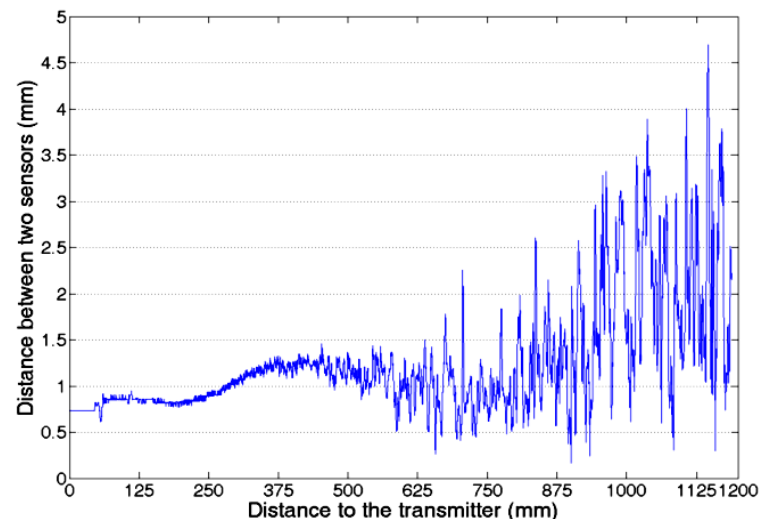

b)

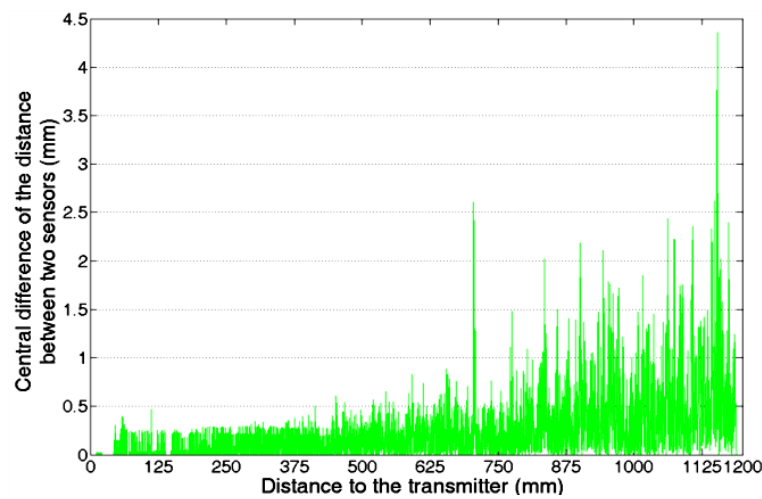

c)

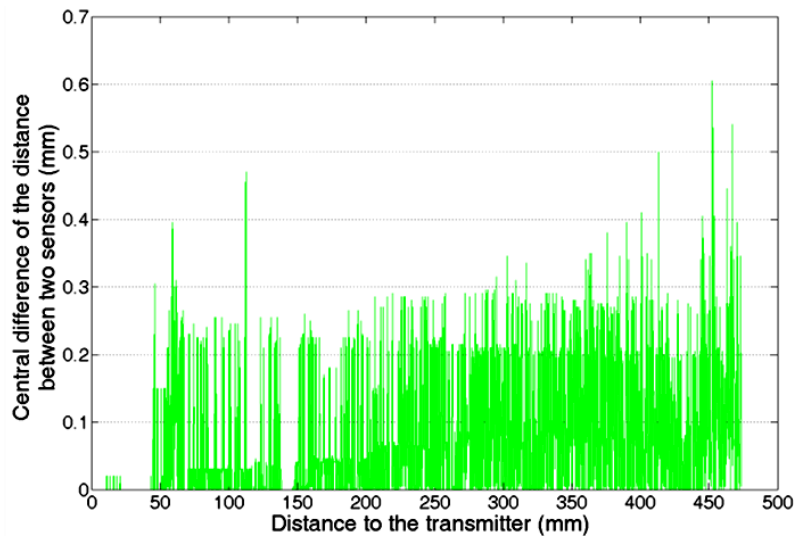

d)

Fig. 3. Dynamic mesaurement. a), b) The distance between two sensors versus the distance between the sensors and the transmitter. c), d) The variation based on finite difference.

\section{CONCLUSION}

In this paper, we present two methods for evaluating the working range of an electromagnetic tracking system 
resulting from the possible mutual interference from two sensors in close proximity. The static measurement required the sensor to stay at specific positions to collect data, and the dynamic measurement evaluated the range during the motion of the sensors. This approach was more flexible at the cost of an additional sensor but was less sensitive for a shorter range. In summary, the first method was more accurate, but it had limited flexibility. The second method was closer to the actual situation in a clinical environment, and it was easier to conduct. In the near future, we plan to conduct additional research aimed at improving the second method.

\section{REFERENCES}

[1] H. Hastenteufel, M. Vetter, H. P. Meinzer, and I. Wolf, "Effect of 3D Ultrasound Probes on the Accuracy of Electromagnetic Tracking Systems," Ultrasound in Medicine and Biology, vol. 32, no. 9, pp. 1359-1368, 2006.

[2] L. Thomas, S. Vijayan, A. Rethy, C. Våpenstad, O. V. Solberg, R. Mårvik, G. Johnsen, and T. N. Hernes, "Navigated laparoscopic ultrasound in abdominal soft tissue surgery: technological overview and perspectives," International Journal of Computer Assisted Radiology and Surgery, vol. 7, no. 4, pp. 585-599, 2012.

[3] K. Schicho, M. Fig, M. Donat, W. Birkfellner, R. Seemann, A. Wagner, H. Bergmann, and R. Ewers, "Stability of Miniature Electromagnetic Tracking Systems," Phys. Med. Biol., vol. 50, pp. 2089-2098, 2005.

[4] Z. Yaniv, E. Wilson, D. Lindisch, and K. Cleary, "Electromagnetic tracking in the clinical environment," Med. Phys., vol. 36, 2009.

[5] J. B. Hummel, M. R. Bax, M. L. Fig, Y. Kang, C. Maurer, W. W. Birkfellner, H. Bergmann, and R. Shahidi, "Design and Application of an Assessment Protocol for Electromagnetic Tracking Systems," Med Phys., vol. 32, no. 7, pp. 2371-2379, 2005.

[6] L. Maier-Hein, A. M. Franz, W. Birkfellner, J. Hummel, I. Gergel, I. Wegner, and H.-P. Meinzer, "Standardized assessment of new electromagnetic field generators in an interventional radiology setting," Med. Phys., vol. 39, pp. 3424, 2012.

[7] A. M. Franz, K. März, J. Hummel, W. Birkfellner, R. Bendl, S. Delorme, H.-P. Schlemmer, H.-P. Meinzer, and L. Maier-Hein, "Electromagnetic tracking for US-guided interventions: standardized assessment of a new compact field generator," International Journal of Computer Assisted Radiology and Surgery, vol. 7, no. 6, pp. 813-818, 2012.

[8] C. Nafis, V. Jensen and R. V. Jako, "Method for evaluating compatibility of commercial electromagnetic (EM) microsensor tracking systems with surgical and imaging tables," in Proc. SPIE,Medical Imaging: Visualization, Image-guided Procedures, and Modeling, vol. 6918, pp. 691820-1 -691820-15, 2008

[9] A. D. Wiles, J. Moore, C. A.Linte, C. Wedlake, A. Ahmad, and T. M. Peters, "Object identification accuracy under ultrasound enhanced virtual reality for minimally invasive cardiac surgery," in Proc. SPIE,
Medical Imaging: Visualization, Image-guided Procedures, and Modeling, vol. 6918, pp. 69180E-1-69180E-12, 2008.

[10] D. M. Kwartowitz, M. E. Rettmann, D. R. Holmes III, and R. A. Robb, "A novel technique for analysis of accuracy of magnetic tracking systems used in image guided surgery," in Proc. SPIE, Medical Imaging: Visualization, Image-Guided Procedures, and Modeling, vol. 7625, pp. 76251L-1-76251L-8, 2010.

[11] L. E. Bø, H. O. Leira, G. A. Tangen, E. F. Hofstad, T. Amundsen, and T. Lang $\varnothing$, "Accuracy of electromagnetic tracking with a prototype field generator in an interventional OR setting," Med. Phys., vol. 39, pp. 399-406, 2012.

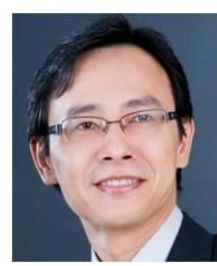

Chia-Hsiang Wu received the BS degree in mathematics from Chung Yuan Christian University in 1996, and the MS and PhD degrees in Computer Science and Information Engineering from National Cheng Kung University in 1998 and 2007, respectively. Currently, he is an Assistant Professor in the Department of Biomedical Engineer in gat I-Shou University, Taiwan. His current research interests include medical image analysis, computer vision, and surgical simulation systems.

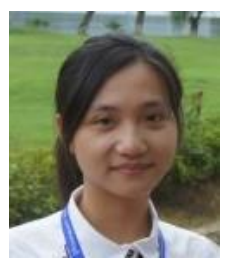

Mei Yun Su was born in Chiayi, Taiwan. She received the BS degree in Biomedical Engineering from I-Shou University, Taiwan, in 2012 and is currently working toward the Master's degree at I-Shou. Her research interests include image/signal processing and calibration procedure for magnetic tracking systems. She is a student member of IEEE.

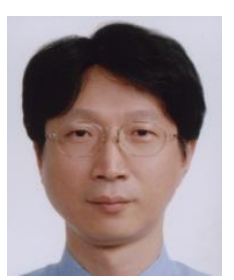

Chien-Chen Chang received the M.D. degree from Chung Shan Medical University, Taichung, Taiwan, R.O.C., in 1984.He is an urologist at the Urological Department, Sin-Lau Hospital, Taiwan. $\mathrm{He}$ is interested in medical engineering to develop better medical equipment to help patients.

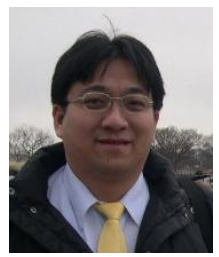

Ting-Sheng Lin was born in Taipei, Taiwan. Ting-Sheng Lin received his B.S. (1997) in Mechanical Engineering, M.S. (1999) and Ph.D. (2004) in Biomedical Engineering from National Cheng Kung University (NCKU), Taiwan. Since 2004,Ting-Sheng has been an Assistant Professor in the Department of Biomedical Engineering, I-Shou University, Kaohsiung, Taiwan. His current research interests are dental and orthopedic biomechanics, computer-aided engineering, and design of medical and surgical instruments.Prof. Lin is also the member of International Society of Biomechanics (ISB) and The International Association for Dental Research (IADR). 